\title{
Interação espacial entre os investimentos no PRONAF e o Índice de Desenvolvimento Rural nos municípios do Nordeste
}

\author{
Spatial interaction between the investments in PRONAF and the Rural \\ Development Index in Northeastern municipalities
}

\author{
Interacción espacial entre las inversiones en el PRONAF y el Índice de Desarrollo \\ Rural en los municipios del Nordeste
}

\author{
Geizibel Lopes Rodrigues ${ }^{1}$ \\ Diego Firmino Costa da Silva ${ }^{1}$
}

Recebido em: 20/12/2018; revisado e aprovado em: 19/06/2019; aceito em: 25/07/2019 DOI: http://dx.doi.org/10.20435/inter.v22i2.2325

\begin{abstract}
Resumo: O objetivo deste trabalho é analisar a interação espacial entre o Índice de Desenvolvimento Rural (IDR) e os investimentos destinados ao PRONAF nos municípios do Nordeste, em 2000 e 2010. Considerandose as relações intermunicipais que podem gerar dependência espacial adotou-se como metodologia a Análise Exploratória de Dados Espaciais (AEDE) bivariada. Foi calculado o Índice Global de Moran para verificar a existência e o grau de correlação espacial das variáveis IDR e PRONAF e o Índice Local de Moran para localizar as aglomerações espaciais. Na análise global os resultados apresentaram correlação positiva para as duas variáveis nos dois períodos, ou seja, uma predominância de aglomerações com valores semelhantes, sejam eles mais baixos ou mais elevados. Na análise local predominaram as aglomerações de municípios BaixoBaixo em que municípios com IDR abaixo da média são vizinhos de municípios com PRONAF também abaixo da média. A existência de dependência espacial dos municípios, tanto quando se olha para as variáveis separadamente, quanto ao analisar as duas variáveis juntas sugere que as características dos municípios exercem, a partir de suas relações intermunicipais, alguma influência nos vizinhos considerando sua relação de proximidade. Além disso, um maior número de municípios em aglomerações Baixo-Baixo mostra que o panorama do desenvolvimento rural no Nordeste ainda é crítico.
\end{abstract}

Palavras-chave: desenvolvimento rural; agricultura familiar; dependência espacial.

Abstract: The objective of this paper is to analyze the spatial interaction between the Rural Development Index (RDI) and the investments destined to PRONAF in the municipalities of the Northeast in 2000 and 2010. Considering the inter-municipal relationships that can generate spatial dependence, the methodology adopted was the Bivariate Exploratory Spatial Data Analysis (AEDE). The Moran Global Index was calculated to verify the existence and degree of spatial correlation of the IDR and PRONAF variables and the Moran Local Index to locate the spatial agglomerations. In the global analysis, the results showed a positive correlation for the two variables in the two periods, that is, a predominance of clusters with similar values, whether they were lower or higher. In the local analysis, agglomerations of Low-Low municipalities predominated, in which municipalities with a RDI below the average are neighbors with municipalities with PRONAF also below the average. The existence of spatial dependence of the municipalities, both when looking at the variables separately, and when analyzing the two variables together, suggests that the characteristics of the municipalities exert, from their inter-municipal relationships, some influence on their neighbors considering their proximity relationship. In addition, a greater number of municipalities in Baixo-Baixo agglomerations shows that the panorama of rural development in the Northeast is still critical.

Keywords: rural development; family agriculture; space dependence.

Resumen: El objetivo de este trabajo es analizar la interacción espacial entre el Índice de Desarrollo Rural (IDI) y las inversiones destinadas al PRONAF en los municipios del Nordeste en 2000 y 2010. Considerando las relaciones intermunicipales que pueden generar dependencia espacial, la metodología se adoptó el Análisis Bivariante Exploratorio de Datos Espaciales (AEDE). Se calculó el Índice Global de Moran para verificar la existencia y grado de correlación espacial de las variables IDR y PRONAF y el Índice Local de Moran para localizar las aglomeraciones espaciales. En el análisis global, los resultados arrojaron una correlación positiva para las dos variables en los dos períodos, es decir, un predominio de conglomerados con valores similares, ya fueran menores o mayores. En el análisis local predominaron las aglomeraciones de municipios Bajo-Bajo, en los que los municipios con una IDR por debajo de la media son vecinos con municipios con PRONAF también

\footnotetext{
${ }^{1}$ Universidade Federal Rural de Pernambuco (UFRPE), Dois Irmãos, Recife, Brasil.
} 
por debajo de la media. La existencia de dependencia espacial de los municipios, tanto al considerar las variables por separado, como al analizar las dos variables en conjunto, sugiere que las características de los municipios ejercen, desde sus relaciones intermunicipales, cierta influencia sobre sus vecinos considerando su relación de proximidad. Además, un mayor número de municipios en las aglomeraciones Baixo-Baixo muestra que el panorama de desarrollo rural en el Nordeste sigue siendo crítico.

Palabras clave: desarrollo rural; agricultura familiar; dependencia espacial.

\section{INTRODUÇÃO}

Dos mais de 5,5 bilhões de pessoas que vivem em países em desenvolvimento, em torno de 3 bilhões residem em áreas rurais, e a agricultura é a principal fonte de subsistência para 86\% dessas famílias (DETHIER; EFFENBERGER, 2012). Neste sentido, é importante analisar como o setor da agricultura familiar tem desempenhado seu papel na promoção do desenvolvimento de um país.

Segundo o relatório da Organização para a Cooperação e Desenvolvimento Econômico (OCDE, 2016), subsidiar o desenvolvimento rural nos países em desenvolvimento é essencial para promover o bem-estar de muitas populações rurais pobres que tendem a continuar crescendo. Daí a importância das políticas públicas voltadas para o desenvolvimento do meio rural como forma de melhoria da qualidade de vida dos agricultores mais pobres e promoção da diminuição das desigualdades sociais.

No que diz respeito ao Brasil, de acordo com o relatório do Comitê da Oxford de Combate à Fome (OXFAM, 2017), o país está entre os oito mais desiguais do mundo e necessita voltar sua atenção principalmente para as áreas rurais onde a pobreza prevalece. De acordo com Vieira Filho (2013), cerca de $66,4 \%$ da população rural brasileira vive em situação de extrema pobreza.

Esse cenário pode indicar que, apesar de haver uma cadeia de políticas voltadas para o meio rural, criadas nas últimas décadas, existe ainda um sério problema a ser resolvido no país quanto à pobreza no campo, o que traz questionamentos acerca da contribuição dessas políticas para o desenvolvimento rural. Uma das políticas mais importantes criadas com objetivo de promover o desenvolvimento rural no Brasil é o Programa de Fortalecimento da Agricultura Familiar (PRONAF), criado em 1996, com objetivo principal de promover o desenvolvimento sustentável da agricultura familiar.

Quanto ao desenvolvimento rural, Corrêa, Silva e Neder (2008), utilizando um índice de Desenvolvimento Rural (IDR), encontraram níveis baixos de IDR nos municípios do Nordeste em comparação com os municípios da região Sul. Além disso, Stege e Parré (2011) identificaram $37 \%$ das microrregiões do país com médio e baixo desenvolvimento rural. De forma geral, tanto os estudos relacionados ao IDR como aqueles referentes ao PRONAF apresentam resultados diversos nos vários contextos do Brasil. Além disso, há uma escassez de trabalhos observando as duas variáveis conjuntamente e, principalmente, utilizando um índice multidimensional como o IDR, que busca uma visão mais ampla dos aspectos do desenvolvimento rural. Não foram identificadas também pesquisas comparando as duas variáveis, considerando um contexto no qual as regiões, estados ou municípios estabelecem constantemente relações entre si, a partir da interação de pessoas e organizações e na troca de informações entre elas, assim como pelo fluxo de renda, pessoas, bens e serviços.

De acordo com Almeida (2004), as relações formais ou informais estabelecidas entre municípios, que, por sua vez, vão além dos limites territoriais, podem gerar um efeito de 
multiplicação, sendo capaz de criar uma dependência espacial entre essas localidades e sua vizinhança. Ou seja, é possível inferir que esses elos de ligação ou a falta deles podem influenciar as condições de acesso às políticas públicas de desenvolvimento rural e o próprio nível de desenvolvimento dos municípios e seus vizinhos. Esse cenário pode ser visualizado em pesquisas como a de Silva, Farias Souza e Silva (2013), em que os autores analisaram a dependência espacial da extrema pobreza nos municípios do Nordeste e observaram, por exemplo, que os municípios com altas taxas de extrema pobreza ou baixas taxas de extrema pobreza estavam exatamente cercados por vizinhos com características semelhantes, fossem eles do mesmo estado ou não. Resultado semelhante pode ser visto no trabalho de Lins et al. (2015), que fizeram uma análise espacial da evolução do Índice de Desenvolvimento Humano nos municípios do Nordeste, verificando que as localidades com baixo IDHM e elevado IDHM estavam próximas de localidades semelhantes.

De acordo com Basile (2008), as pesquisas empíricas vêm considerando fortemente o papel dos efeitos espaciais entre regiões, ou seja, a existência de dependência espacial em seu processo de crescimento econômico, pois as taxas de crescimento do PIB per capita de uma economia depende criticamente do crescimento das economias próximas, e não apenas de suas próprias condições. Lesage e Fischer (2008) afirmam que, apesar das análises convencionais de crescimento assumirem que as observações regionais são independentes, há um consenso crescente de que as taxas de crescimento da renda regional sofrem de dependência espacial.

Nesse contexto, o objetivo deste trabalho é analisar a interação espacial entre o Índice de Desenvolvimento Rural (IDR) e os investimentos destinados ao PRONAF nos municípios do Nordeste. Para isso, será calculado o IDR para cada município e verificada a existência de correlação espacial entre o IDR e a distribuição dos recursos do PRONAF para os anos de 2000 e 2010.

Um dos aspectos importantes deste trabalho, além do interesse na relação das políticas públicas rurais com o desenvolvimento rural, é a utilização de uma abordagem que permite o estudo da relação entre variáveis, neste caso, o IDR e a distribuição dos recursos do PRONAF, considerando a localização espacial das unidades de análise, a fim de verificar a possível existência de dependência espacial entre elas.

Assim, para apoiar este estudo, incluindo esta breve introdução, apresenta-se a seguir, na seção 2, uma breve explanação acerca do desenvolvimento rural e da agricultura familiar; na seção 3, são apresentados os procedimentos metodológicos e dados; e, na seção 4, os resultados do trabalho. Por fim, apresentam-se na seção 5 as considerações finais.

\section{DESENVOLVIMENTO RURAL E AGRICULTURA FAMILIAR}

De acordo com Hill, Goll e Karlsson (2007), é importante pensar o conceito de desenvolvimento rural como uma questão que deixou de ser apenas setorial, tendo a agricultura como único foco, para se tornar um conceito que abrange uma gama de questões, envolvidas no âmbito do desenvolvimento, como questões demográficas, econômicas, sociais e ambientais. Além disso, a ideia do desenvolvimento rural sempre esteve, em grande parte, ligada à pobreza no meio rural, que é evidente em todas as regiões do mundo que possuem dados disponíveis sobre esse problema, mas, principalmente, nos países em desenvolvimento, como os da América Latina, onde cerca de $60 \%$ da população pobre se encontra no meio rural (RODRÍGUEZ-POSE; HARDY, 2015). 
No entanto, nos últimos anos, o meio rural tornou-se importante para a redução da pobreza e as atenções de organizações se voltaram para a agricultura pela urgente necessidade de alimentar a população mundial crescente, prevista para mais de 9 bilhões até 2050, e pela necessidade de pensar em como alimentar a população pobre que fará parte desse contingente. Nessa conjuntura, em que a agricultura vem sendo vista como estratégia para o desenvolvimento rural, houve um aumento na construção de políticas públicas para o setor e na necessidade de entender tanto a atuação dessas políticas quanto sua recepção pelo ambiente rural. Por essa razão, organizações e pesquisadores trabalham na construção de importantes indicadores para medir o desenvolvimento no meio rural.

Determinadas pesquisas que utilizaram algum Índice de Desenvolvimento Rural (IDR) mostraram que os menores índices se encontram, principalmente, em regiões mais afastadas dos centros urbanos e com pior infraestrutura local. Por exemplo, Michalek e Zarnekow (2011) construíram um índice multidimensional para medir o nível de desenvolvimento rural em duas regiões da União Europeia, e Tae-Hwa e Seung-Ryong (2016) utilizaram o IDR para analisar o estado e processo do desenvolvimento rural do Vietnã. Resultados semelhantes são vistos nas pesquisas nacionais, como em Melo e Parré (2007), em que os autores calcularam o IDR para os municípios do Paraná; e Stege e Parré (2011), em que analisam o desenvolvimento rural nas microrregiões brasileiras.

Essas pesquisas são importantes para entender o desenvolvimento rural em contextos locais ou globais, o que pode auxiliar no direcionamento de políticas que levem esses contextos em consideração, ou seja, que as políticas de desenvolvimento rural estejam integradas com as medidas necessárias à promoção do desenvolvimento.

\subsection{PRONAF e desenvolvimento na agricultura familiar}

As políticas públicas para o desenvolvimento rural, incluindo as políticas de crédito rural, têm sua importância reconhecida por organizações internacionais, como a Organização das Nações Unidas para a Agricultura e Alimentação (FAO) e a OCDE, as quais enfatizam a relevância da pequena agricultura como um dos principais caminhos para retirada de milhões de pessoas que ainda vivem em condições de pobreza no meio rural.

No Brasil, o PRONAF é uma das políticas mais importantes no sentido de combater a pobreza rural por meio da diminuição das desigualdades no acesso às políticas de crédito. Neste sentido, a expectativa é de que o PRONAF, como um programa de desenvolvimento rural voltado à agricultura familiar, atenda principalmente os pequenos agricultores. De acordo com Vieira Filho (2013), estes agricultores compõem a parcela mais pobre da população, sendo em torno de 2,9 milhões de estabelecimentos à margem da produção agropecuária no Brasil.

No entanto os estudos referentes ao PRONAF, apesar de o abordarem como um dos mais importantes para o desenvolvimento rural, não trazem um consenso sobre os possíveis impactos positivos da política. Por exemplo, Damasceno, Khan e Lima (2011) analisaram a contribuição do PRONAF para o desenvolvimento sustentável da agricultura familiar no estado do Ceará, por meio da relação entre o PRONAF e um Índice de Sustentabilidade (IS), e encontraram baixo nível de sustentabilidade, tanto para beneficiários como não beneficiários, indicando pouca relação do programa com o índice.

Assim, também, Batista e Neder (2014) buscaram os efeitos do PRONAF sobre a pobreza rural no Brasil entre 2001 e 2009. Concluíram que uma elevação em 10\% do crédito do PRONAF 
tende a elevar a renda per capita domiciliar rural em apenas 0,24\% e a reduzir a desigualdade de renda em $0,36 \%$. Porém, de acordo com os autores, o crédito do PRONAF apresenta efeito maior na renda entre os estados mais ricos.

Em pesquisa recente realizada por Araújo e Vieira Filho (2018) sobre os impactos do PRONAF na agricultura entre 2007 e 2016, os autores afirmam que o programa foi, ao longo dos anos, voltado para a modernização do setor agropecuário e ao aprimoramento da utilização de novas tecnologias para a expansão das práticas produtivas, e, com essa atuação, o programa tende a não estimular a diversificação da produção, o que dificultaria a redução da pobreza. Porém fortalecer a capacidade e a diversificação produtiva da agricultura familiar é justamente um dos objetivos do programa, o que contribuiria para a segurança, autonomia e melhoria de renda do agricultor familiar.

Alguns, por sua vez, vêm mostrando resultados positivos em análises mais agregadas e quando verificam o efeito do programa sobre variáveis mais gerais, tal como em Castro, Resende e Pires (2014) e Marioni et al. (2016), que investigaram os efeitos do programa sobre o PIB nacional e setorial. Em contextos menos agregados, Justo e Lima (2016) investigaram a importância do programa sobre um índice de qualidade de vida e Macedo (2017) analisou os efeitos do programa sobre variáveis como valor da produção, valor da produção por hectare e por trabalhador, em contextos específicos de Pernambuco, encontrando resultados positivos sobre a vida de pequenos beneficiários do programa.

No que se refere a estudos relacionando o PRONAF com o desenvolvimento rural utilizando um índice, Silva (2006) faz uma análise de correlação entre o PRONAF crédito e infraestrutura e o IDR nas regiões Nordeste e Sul para 2000. Os resultados mostraram correlação positiva entre o PRONAF e o IDR para o Nordeste, e uma correlação negativa para o Sul. De acordo com Silva (2006), em regra, para as duas regiões, os recursos tenderam a privilegiar os municípios com IDR médio e alto, sugerindo que os municípios que acessaram essas linhas de crédito, no período estudado, foram aqueles com IDR mais elevado. Nesse sentido, Fernandes (2011) realizou estudo semelhante para o Nordeste e o Sul em três períodos. No primeiro período, de 2001-2003, constatou que os municípios do Nordeste com IDR baixo ou muito baixo possuíam níveis baixos de captação do PRONAF e assim sucessivamente; no período de 2004-2005, os municípios de IDR baixo evoluíram para uma maior captação e, no período de 2007-2009, os resultados regrediram. Para a região Sul, os três períodos apresentaram um equilíbrio maior na captação dos recursos.

Nas pesquisas apresentadas acima, tanto as que relacionam o programa com variáveis isoladas como as que buscam sua relação com um índice mais amplo já sugerem que existe relação entre o PRONAF e os aspectos do desenvolvimento rural. Porém não se identificaram pesquisas que buscassem analisar essa relação considerando aspectos espaciais. Essas relações não podem ser ignoradas, pois, como afirma Basile (2008), as economias interagem entre si por meio da mobilidade de fatores como migração de capital e trabalho, nas relações comerciais, de difusão tecnológica e transbordamento de conhecimento, gerando dependência espacial entre elas.

Dessa forma, a análise da interação espacial entre o IDR e a distribuição dos recursos do PRONAF, proposta neste trabalho, tem sua contribuição tanto no que diz respeito à análise do programa, relacionando-o a um índice multidimensional, como a utilização de uma abordagem espacial ainda pouco utilizada e que possibilita captar aspectos que podem ser despercebidos por outras abordagens que desconsideram as relações espaciais. 


\section{PROCEDIMENTOS METODOLÓGICOS E DADOS}

Com o objetivo de analisar a interação espacial entre o Índice de Desenvolvimento Rural (IDR) e os investimentos destinados ao PRONAF nos municípios do Nordeste, será realizada uma Análise Exploratória de Dados Espaciais (AEDE), com a utilização do software GeoDa apresentado por Anselin, Syabri e Kho (2006).

\subsection{Os dados}

Os dados utilizados na pesquisa foram os valores dos investimentos anuais do PRONAF para os municípios do Nordeste, disponíveis na plataforma do Ministério do Desenvolvimento Agrário (MDA). Os valores foram deflacionados com base no IPCA e divididos pelo valor populacional dos municípios, para obtenção de um valor per capita e melhor comparação de municípios com valores distintos.

Quanto aos dados para a construção do IDR, foram utilizados os microdados dos Censos Demográficos de 1991, 2000 e 2010. Além destes, foram utilizados dados do Censo Agropecuário de 1996 e 2006 e da Pesquisa Agrícola Municipal disponível no Sistema IBGE de Recuperação Automática (SIDRA), de 2000 e 2010. A tabela final com os dados do PRONAF e IDR ficou composta de 1.786 municípios, após a exclusão do município de Fernando de Noronha.

\subsection{As dimensões do Índice de Desenvolvimento Rural}

Para o cálculo do IDR, tomou-se como base a proposta de Kageyama (2004) e Corrêa, Silva e Neder (2008), que calcularam o IDR para o contexto brasileiro a partir das orientações da Organização para a Cooperação e Desenvolvimento Econômico (OCDE) para a elaboração de Índices de Desenvolvimento Territorial.

Porém algumas modificações foram realizadas na construção do IDR para a presente pesquisa: para o indicador de bem-estar social, foi calculada uma taxa de alfabetização em vez de taxa de analfabetismo. Para o indicador de meio ambiente, foi calculada a proporção de estabelecimentos que não usam adubos fertilizantes em vez da proporção de estabelecimentos que os utilizam; além disso, foi substituída a variável ausência de monocultura por um índice de diversificação agrícola e, por fim, para o cálculo final do IDR, adotou-se a média geométrica que considera o peso de cada indicador, em substituição à média simples utilizada por Kageyama (2004) e Corrêa, Silva e Neder (2008). A seguir, são apresentados no Quadro 1 os indicadores e as variáveis que compõem o IDR, fórmulas de cálculo e fontes de dados utilizadas. 
Quadro 1 - Indicadores e variáveis selecionadas para o IDR

\begin{tabular}{|c|c|c|c|}
\hline Indicador & Descrição da Variável & Cálculo & Base de dados \\
\hline \multirow{4}{*}{$\begin{array}{l}\text { Indicador de } \\
\text { População } \\
(\text { IPOP })= \\
(a+b+c+d) / 4\end{array}$} & $\begin{array}{l}\text { a) Densidade demográfica } \\
\text { (padronizada) }\end{array}$ & $\begin{array}{l}\text { População absoluta/total do } \\
\text { município dividido pela área } \\
\text { territorial }\left(\mathrm{km}^{2}\right)\end{array}$ & $\begin{array}{l}\text { Variável já } \\
\text { calculada pelo } \\
\text { IBGE (disponível } \\
\text { no site) }\end{array}$ \\
\hline & $\begin{array}{l}\text { b) Variação da população } \\
\text { rural (padronizada) }\end{array}$ & $\begin{array}{l}\text { Diferença entre: população rural de } \\
2000 \text { e } 2010 \text {, dividida pela população } \\
\text { rural de } 2000 \text { para o IDR 2010, e } \\
\text { entre } 1991 \text { e } 2000 \text {, dividida pela } \\
\text { população rural de } 1991 \text { para o IDR } \\
2000\end{array}$ & \multirow{3}{*}{$\begin{array}{l}\text { Variáveis } \\
\text { coletadas dos } \\
\text { - Microdados } \\
\text { dos Censos } \\
\text { Demográficos de } \\
2000 \text { e } 2010\end{array}$} \\
\hline & $\begin{array}{l}\text { c) Proporção da população } \\
\text { rural no município }\end{array}$ & $\begin{array}{l}\text { Total da população rural do município } \\
\text { dividido pela sua população total }\end{array}$ & \\
\hline & $\begin{array}{l}\text { d) Proporção da população } \\
\text { rural que não morou } \\
\text { sempre no município } \\
\text { (migração) }\end{array}$ & $\begin{array}{l}\text { População que não morou sempre } \\
\text { no município dividida pela população } \\
\text { rural total }\end{array}$ & \\
\hline \multirow{5}{*}{$\begin{array}{l}\text { Indicador de Bem- } \\
\text { Estar Social (IBES) } \\
=(a+b+c+d+e) / 5\end{array}$} & a) Taxa de alfabetização & $1-\frac{\text { proporção de analfabetos }}{\text { total da população rural }}$ & \multirow{5}{*}{$\begin{array}{l}\text { Variáveis } \\
\text { coletadas dos } \\
\text { Microdados } \\
\text { dos Censos } \\
\text { Demográficos de } \\
2000 \text { e } 2010\end{array}$} \\
\hline & $\begin{array}{l}\text { b) Média de anos de } \\
\text { estudo (padronizada) } \\
\text { nos domićlíios rurais }\end{array}$ & $\begin{array}{l}\text { Anos de estudo da população de } 15 \\
\text { anos ou mais divididos pelo total da } \\
\text { população de idade igual ou superior } \\
\text { a } 15\end{array}$ & \\
\hline & $\begin{array}{l}\text { c) Proporção de crianças de } \\
7 \text { a } 14 \text { anos na escola }\end{array}$ & $\begin{array}{l}\text { Total de crianças rurais de } 7 \text { a } 14 \\
\text { anos na escola dividido pelo total de } \\
\text { crianças rurais de } 7 \text { a } 14 \text { anos }\end{array}$ & \\
\hline & $\begin{array}{l}\text { d) Proporção de domicílios } \\
\text { com água encanada em } \\
\text { pelo menos um cômodo }\end{array}$ & $\begin{array}{l}\text { Total de domicílios rurais com } \\
\text { água encanada em pelo menos } \\
\text { um cômodo dividido pelo total de } \\
\text { domicílios rurais }\end{array}$ & \\
\hline & $\begin{array}{l}\text { e) Proporção de domicílios } \\
\text { com abastecimento de } \\
\text { água }\end{array}$ & $\begin{array}{l}\text { Total de domicílios rurais com } \\
\text { abastecimento de água pela rede de } \\
\text { distribuição dividido pelo total de } \\
\text { domicílios rurais }\end{array}$ & \\
\hline \multirow{2}{*}{$\begin{array}{l}\text { Indicador de } \\
\text { Desenvolvimento } \\
\text { Econômico } \\
(\text { IDE })=(a+b) / 2\end{array}$} & $\begin{array}{l}\text { a) Proporção de ocupados } \\
\text { rurais em atividades não } \\
\text { agrícolas }\end{array}$ & $\begin{array}{l}\text { Relação entre o total de ocupados em } \\
\text { atividades não agrícolas e o total dos } \\
\text { ocupados nos domicílios rurais }\end{array}$ & \multirow{2}{*}{$\begin{array}{l}\text { Variáveis } \\
\text { coletadas dos } \\
\text { Microdados } \\
\text { dos Censos } \\
\text { Demográficos de } \\
2000 \text { e } 2010\end{array}$} \\
\hline & $\begin{array}{l}\text { b) Rendimento médio } \\
\text { domiciliar per capita } \\
\text { (padronizado) }\end{array}$ & $\begin{array}{l}\text { Renda domiciliar total dividida pelo } \\
\text { número de membros do domicílio } \\
\text { (exceto agregados, pensionistas etc.) }\end{array}$ & \\
\hline
\end{tabular}

\footnotetext{
${ }^{2}$ A padronização consiste em fazer uma transformação algébrica para que o índice varie no intervalo de 0 e 1, e essa transformação é o quociente de (valor da variável - mínimo)/(máximo - mínimo).
} 


\begin{tabular}{|c|c|c|c|}
\hline Indicador & Descrição da Variável & Cálculo & Base de dados \\
\hline \multirow{3}{*}{$\begin{array}{l}\text { Indicador de Meio } \\
\text { Ambiente }(I M A)= \\
\qquad(a+b+c) / 3\end{array}$} & $\begin{array}{l}\text { a) Proporção de } \\
\text { estabelecimentos que } \\
\text { adotam práticas de } \\
\text { conservação do solo }\end{array}$ & $\begin{array}{l}\text { Total de estabelecimentos que } \\
\text { adotam práticas de conservação } \\
\text { do solo dividido pelo total de } \\
\text { estabelecimentos agropecuários }\end{array}$ & \multirow{2}{*}{$\begin{array}{l}\text { Dados do Censo } \\
\text { Agropecuário } \\
1996 \text { para o } \\
\text { IDR } 2000 \text { e } \\
\text { dados do Censo } \\
\text { Agropecuário } \\
2006 \text { para o IDR } \\
2010\end{array}$} \\
\hline & $\begin{array}{l}\text { b) Proporção de } \\
\text { estabelecimentos que } \\
\text { não usam adubos e } \\
\text { fertilizantes }\end{array}$ & $1-\frac{\begin{array}{c}\text { total de estabelecimentos que } \\
\text { usam adubosefertilizantes } \\
\text { químicos }\end{array}}{\text { total de estabelecimentos }}$ & \\
\hline & $\begin{array}{l}\text { c) Índice de diversificação } \\
\text { agrícola }^{3}\end{array}$ & $1-\frac{\begin{array}{c}\text { somatório do quadrado da } \\
\text { área de cada cultura }\end{array}}{\text { área de todas as culturas }}$ & $\begin{array}{l}\text { Pesquisa } \\
\text { Agrícola } \\
\text { Municipal/SIDRA } \\
\text { (IBGE), dados de } \\
2000 \text { e } 2010\end{array}$ \\
\hline
\end{tabular}

Fonte: adaptado de Kageyama (2004) e Corrêa, Silva e Neder (2008).

O primeiro indicador é o Indicador de População (IPOP), que trata das características populacionais de cada município. As variáveis desse indicador buscam captar a capacidade dos municípios em reter ou atrair pessoas, pois entende-se, por exemplo, que uma maior densidade demográfica pode ser indício de maior atratividade do município (KAGEYAMA, 2004).

O segundo indicador é o Indicador de Bem-Estar Social (IBES), que se refere às condições do domicílio em relação à educação e acesso à água. Essas duas variáveis são importantes para a melhoria da qualidade de vida da população pobre rural, pois a deficiência na educação formal gera, entre outros problemas, dificuldades ao desenvolvimento rural, podendo contribuir para o baixo dinamismo de uma localidade e, consequentemente, à minimização da carência de capacidade de absorção tecnológica (VIEIRA FILHO, 2013).

O terceiro indicador é o Indicador de Desenvolvimento Econômico (IDE), que busca captar as condições de rendimento familiar e que pode trazer informações sobre a distribuição da renda rural e a proporção de ocupados em atividades não agrícolas, pois, de acordo com Corrêa, Silva e Neder (2008), as famílias que são exclusivamente agrícolas são as que recebem menores rendimentos e, considerando estes aspectos, quanto maior for o número de ocupados em atividades não agrícolas, maior o Desenvolvimento Rural, devido à diversidade de atividades que podem melhorar a renda familiar.

Por fim, tem-se o indicador individual IMA (Indicador de Meio Ambiente), que busca a relação dos estabelecimentos rurais com o ambiente. Tendo em vista os recursos naturais, principalmente o solo, tem uma vida útil de produção. É importante saber se os estabelecimentos estão tendo algum tipo de cuidado para preservação desse recurso essencial à produção agrícola.

Criadas as variáveis e feito o cálculo dos indicadores individuais, pode-se então calcular o IDR para os municípios, utilizando a média geométrica como mostrado a seguir. A escolha da média geométrica se dá pela sua capacidade de balancear o peso dos indicadores, de forma que o valor de um indicador muito baixo não seja compensado por outro.

$$
I D R=\sqrt[4]{(i p o p * i b e s * i d e * i m a)}
$$

\footnotetext{
${ }^{3}$ Calculado por meio do Índice de Diversificação de Simpson que assume o valor 0 no caso de monoculturas e se aproxima de 1 com o aumento da diversidade da produção agrícola.
} 
Finalmente, com o IDR e valores do PRONAF por município, é possível então fazer a análise exploratória dos dados espaciais.

\subsection{A análise exploratória de dados espaciais}

A análise exploratória de dados espaciais (AEDE) pode ser definida como um conjunto de técnicas que permite descrever distribuições espaciais, assim como verificar a existência de padrões espaciais, clusters e locais atípicos espaciais, outliers (ANSELIN; SYABRI; SMIRNOV, 2002). Por meio da AEDE, é possível verificar a existência de autocorrelação espacial, que pode ser medida tanto em termos globais como locais. Em termos globais, pode ser calculada por meio do Índice Global de Moran, que mostra o grau de associação espacial para todo o conjunto de dados, como representado na Equação 2:

$$
I=\frac{n}{\sum_{i=1}^{n} \sum_{j=1}^{n} w_{i j}} \frac{\sum_{i=1}^{n} \sum_{j=1}^{n} w_{i j}\left(y_{i}-\bar{y}\right)\left(y_{j}-\bar{y}\right)}{\sum_{i=1}^{n}\left(y_{i}-\bar{y}\right)^{2}}
$$

Em que n é o número de unidades espaciais observadas, o $y_{i}$ é a variável de interesse e $y$ é a média dessa variável. $O w_{i j}$ é o elemento da matriz de pesos espaciais chamada de $w$, que é previamente definida para o par de unidades espaciais $i$ e $j$. A definição de uma matriz de vizinhança é necessária para que seja possível a identificação dessas potenciais correlações espaciais.

As matrizes de vizinhança podem ser definidas por contiguidade, em que se define vizinhança de primeira ou segunda ordem, e as matrizes de contiguidade mais comuns são os modelos da rainha (Queen Contiguity) e da torre (Rook Contiguity), que imitam os movimentos das peças no tabuleiro de xadrez e, também, podem ser definidas por distância com K-vizinhos mais próximos.

Após a definição da matriz de vizinhança, é possível calcular o I de Moran Global, que pode ser visualizado em um gráfico conhecido como gráfico de espalhamento de Moran, o qual é dividido em quatro quadrantes que representam as categorias de associação espacial Alto-Alto (AA) e Baixo-Baixo (BB), Alto-Baixo (AB) e Baixo-Alto (BA). O Índice varia entre-1 e 1 e, quanto mais próximo de zero, menor a autocorrelação espacial.

Em termos locais, o Índice de Moran observa a existência de autocorrelação espacial entre o valor observado de uma variável em determinada localidade e o valor observado em localidades vizinhas, permitindo a localização dos clusters e outliers e sua significância estatística. O cálculo do I de Moran Local segue Equação 3.

$$
I_{(i)}=\frac{\left(y_{i}-\bar{y}\right) \sum_{j=1}^{n}\left(y_{j}-\bar{y}\right)}{\sum_{i=1}^{n}\left(y_{i}-\bar{y}\right)^{2}}
$$

Porém, como esta proposta de pesquisa trabalha com duas variáveis, os valores do IDR e os recursos do PRONAF, e pretende-se analisar a relação espacial entre elas conjuntamente, a análise exploratória de dados espaciais pode ser aplicada também a esse tipo de estudo tanto para o índice global como o local de Moran, por meio de uma abordagem bivariada.

O Índice de Moran Global bivariado quantifica a provável dependência espacial entre duas variáveis, ou seja, o grau de associação espacial entre uma variável (zk) observada numa dada localização com outra variável (zl) observada nas localizações "vizinhas" (ANSELIN; SYABRI; SMIRNOV, 2002). O cálculo pode ser descrito como: 


$$
I_{k l}=\frac{z_{k} w z_{l}}{n}
$$

Em que $n$ é o número de observações, $w$ representa a matriz de vizinhança estabelecida e $k$ e $l$ são as variáveis de interesse. Já o $I$ de Moran Local bivariado pode ser dado da seguinte forma:

$$
I_{k l}^{i}=z_{k}^{i} \sum_{j} w_{i j} z_{l}^{j}
$$

Em que é $I_{k l}^{i}$ o LISA (Indicadores Locais de Associação Espacial) bivariado, $z_{k}^{i}$ é a primeira variável a ser considerada, $z_{l}^{j}$ a segunda variável e $w_{i j}$ é o elemento da matriz de pesos espaciais chamada de $w$. O cálculo dos índices global e local de Moran bivariados foram realizados.

\section{RESULTADOS}

Antes de iniciar a apresentação dos resultados da análise espacial, entre o IDR e os recursos do PRONAF, cabe mostrar algumas informações descritivas em relação às variáveis. No Quadro 2, pode ser observada uma primeira estatística sobre o IDR para os anos de 2000 e 2010. O quadro mostra a diferença de cálculo do IDR pela média geométrica (MG) proposta neste trabalho, comparada à média aritmética (MA) proposta por Kageyama (2004) e Corrêa, Silva e Neder (2008).

Quadro 2 - Comparação entre os IDRs de 2000 e 2010

\begin{tabular}{c|c|c|c|c|c}
\hline \multicolumn{3}{|c|}{ IDR 2000 } & \multicolumn{3}{c}{ IDR 2010 } \\
\hline & MG & MA & & MG & MA \\
\hline Mínimo & 0,1051 & 0,1410 & Mínimo & 0 & 0,0834 \\
\hline Máximo & 0,6096 & 0,6255 & Máximo & 0,5116 & 0,5895 \\
\hline Média & 0,2665 & 0,2854 & Média & 0,3020 & 0,3812 \\
\hline Desvio-Padrão & 0,0518 & 0,0545 & Desvio-Padrão & 0,0571 & 0,0574 \\
\hline
\end{tabular}

Fonte: Elaboração própria, a partir do cálculo do IDR.

Em primeiro lugar, é possível perceber que o IDR calculado pela MG tem valor menor que calculado pela MA, porém a média geométrica foi escolhida para apresentar os resultados por ser capaz de balancear o peso dos indicadores, de forma que o valor de um indicador não compense outro. Como é possível observar, utilizando a média geométrica, a variação da média do IDR entre 2000 e 2010 foi menor do que se fosse utilizada a média aritmética. Esse primeiro resultado indica que os componentes do IDR variaram em sentidos diferentes, ou seja, enquanto alguns indicadores melhoraram, outros pioraram. Apesar disso, em média, houve um aumento do IDR para os municípios da região Nordeste, de 2000 para 2010. Adicionalmente, o desviopadrão de 0,05 em todos os casos indica pouca variação em relação à média.

Ainda em relação ao IDR, segue abaixo, nas Figuras 1 e 2, mapas de quantil, divididos em quatro categorias (quartil) para classificação do IDR e visualização da distribuição espacial do índice para os municípios do Nordeste. Municípios com valores abaixo do primeiro quartil foram classificados como muito baixo; valores localizados entre o primeiro quartil e a mediana, baixo; valores entre a mediana o terceiro quartil, médio; e valores acima do valor do terceiro quartil, alto. 
Figuras 1 e 2 - Mapas de Quartil para os IDRs 2000 e 2010
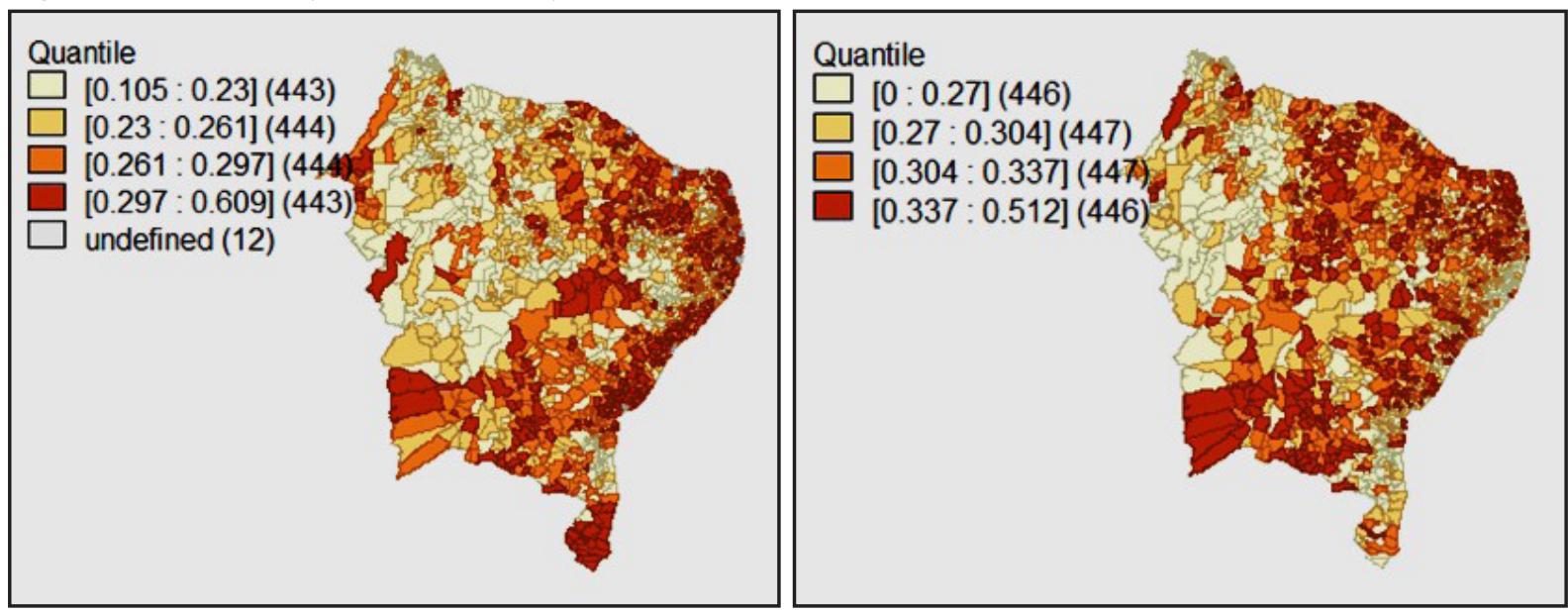

Fonte: Elaboração própria, a partir dos dados do MDA.

Observando o comportamento do índice em relação à distribuição espacial, em 2000, o IDR foi mais elevado nos municípios mais próximos do litoral e nota-se que havia mais aglomerados nas bordas do mapa, em torno das capitais. Em 2010, esses aglomerados diminuíram em torno da maioria das capitais e se espalharam em direção ao interior do mapa. Em 2010, é possível notar um aglomerado no oeste baiano bem mais concentrado que em 2000. Stege e Parré (2011), ao analisarem o IDR para as microrregiões do Brasil, apontaram também o oeste baiano como uma das microrregiões com IDR mais elevado e uma das áreas que vem mostrando maior dinamismo na região em relação à produção agrícola, principalmente grãos para exportação. Outro aglomerado com IDR acima do terceiro quartil em 2000 aglomera alguns municípios da região do São Francisco, entre eles, Petrolina, Lagoa Grande e Santa Maria da Boa Vista, em Pernambuco; e Juazeiro, Curaçá e Sobradinho, na Bahia, que, por sua vez, apresentaram valores abaixo do terceiro quartil em 2010.

Em relação aos dados do PRONAF, na Tabela 1, observam-se informações sobre os valores médios e os respectivos desvios-padrão para a distribuição dos recursos do programa entre 2000 e 2010, calculados para todos os municípios do Nordeste. O valor médio da distribuição do PRONAF por município aumenta em direção ao ano de 2010, mas o desvio-padrão segue a mesma tendência de crescimento. Essa primeira análise pode indicar que, em geral, o investimento médio no PRONAF cresceu nos municípios nordestinos. Porém, tendo em vista o aumento no desvio-padrão, é possível inferir que as disparidades nos valores acessados pelos municípios cresceram também.

Tabela 1 - Valores médios do PRONAF nos municípios do Nordeste

\begin{tabular}{c|c|c}
\hline Ano & Média (R\$) & Desvio-Padrão (R\$) \\
\hline 2000 & 24,70022 & 44,18223 \\
\hline 2001 & 22,07315 & 46,22764 \\
\hline 2002 & 18,22532 & 35,79707 \\
\hline 2003 & 18,78864 & 35,55389 \\
\hline 2004 & 33,32179 & 53,71982 \\
\hline 2005 & 47,40908 & 62,65326 \\
\hline 2006 & 83,82275 & 93,05562 \\
\hline
\end{tabular}




\begin{tabular}{c|c|c}
\hline Ano & Média (R\$) & Desvio-Padrão (R\$) \\
\hline 2007 & 72,29074 & 81,07046 \\
\hline 2008 & 50,71265 & 60,55127 \\
\hline 2009 & 45,48917 & 56,10808 \\
\hline 2010 & 53,59047 & 57,03772 \\
\hline
\end{tabular}

Fonte: Elaboração própria, a partir dos dados do MDA.

Entre os anos de 2000 e 2010, 2006 foi o ano em que o Nordeste teve seu maior valor médio em participação no PRONAF. De acordo com Grisa, Junior e Buchweitz (2014), a participação maior do Nordeste nesse ano se deve em grande parte às críticas que o programa vinha sofrendo em relação à concentração dos recursos e contratos na região Sul, a qual obteve aproximadamente $60 \%$ dos recursos totais em 1997 e 2000. Ainda de acordo com Grisa, Junior e Buchweitz (2014), muitas mudanças foram implementadas no programa a partir de 2002, como a flexibilização das regras financeiras, mudanças institucionais no sentido de diversificar o público da agricultura familiar, a diversificação das atividades econômicas apoiadas e a simplificação das condições de acesso. Mudanças que podem ter contribuído para uma diminuição do viés concentrador já apontado.

\subsection{Análise a partir do Índice de Moran Global Bivariado}

A seguir, são apresentados os resultados alcançados a partir da análise espacial. Inicialmente, é apresentado o resultado do I de Moran Global Bivariado, calculado para verificar a existência de correlação espacial entre o IDR e a distribuição dos recursos do PRONAF. O índice foi calculado para cada ano do estudo, considerando a matriz de vizinhança de contiguidade Queen. Esta matriz estabelece que os municípios são considerados vizinhos entre si e compartilham da mesma fronteira.

Os gráficos de dispersão de Moran bivariado indicam a existência de quatro possíveis situações na relação espacial entre as variáveis IDR e recursos do PRONAF. O quadrante superior direito (Q1) mostra os agrupamentos Alto-Alto (AA), em que os municípios (unidades espaciais) que possuem valores altos do IDR são vizinhos de municípios que possuem valores altos do PRONAF; o quadrante inferior esquerdo (Q2) mostra os agrupamentos Baixo-Baixo (BB), em que os municípios com baixos valores do IDR são vizinhos de municípios com baixos valores do PRONAF; já o quadrante superior esquerdo (Q3) mostra os agrupamentos Baixo-Alto (BA), em que os municípios com valores baixos do IDR são vizinhos de municípios com valores altos do PRONAF; e o quadrante inferior direito (Q4) mostra os agrupamentos Alto-Baixo (AB), em que municípios com valores altos do IDR são vizinhos de municípios com valores altos do PRONAF. $O$ I de Moran Global Bivariado é apresentado nas Figuras 3 e 4, por meio dos Gráficos de Dispersão de Moran. 
Figuras 3 e 4 - Gráficos de Dispersão de Moran Bivariados para IDR e PRONAF
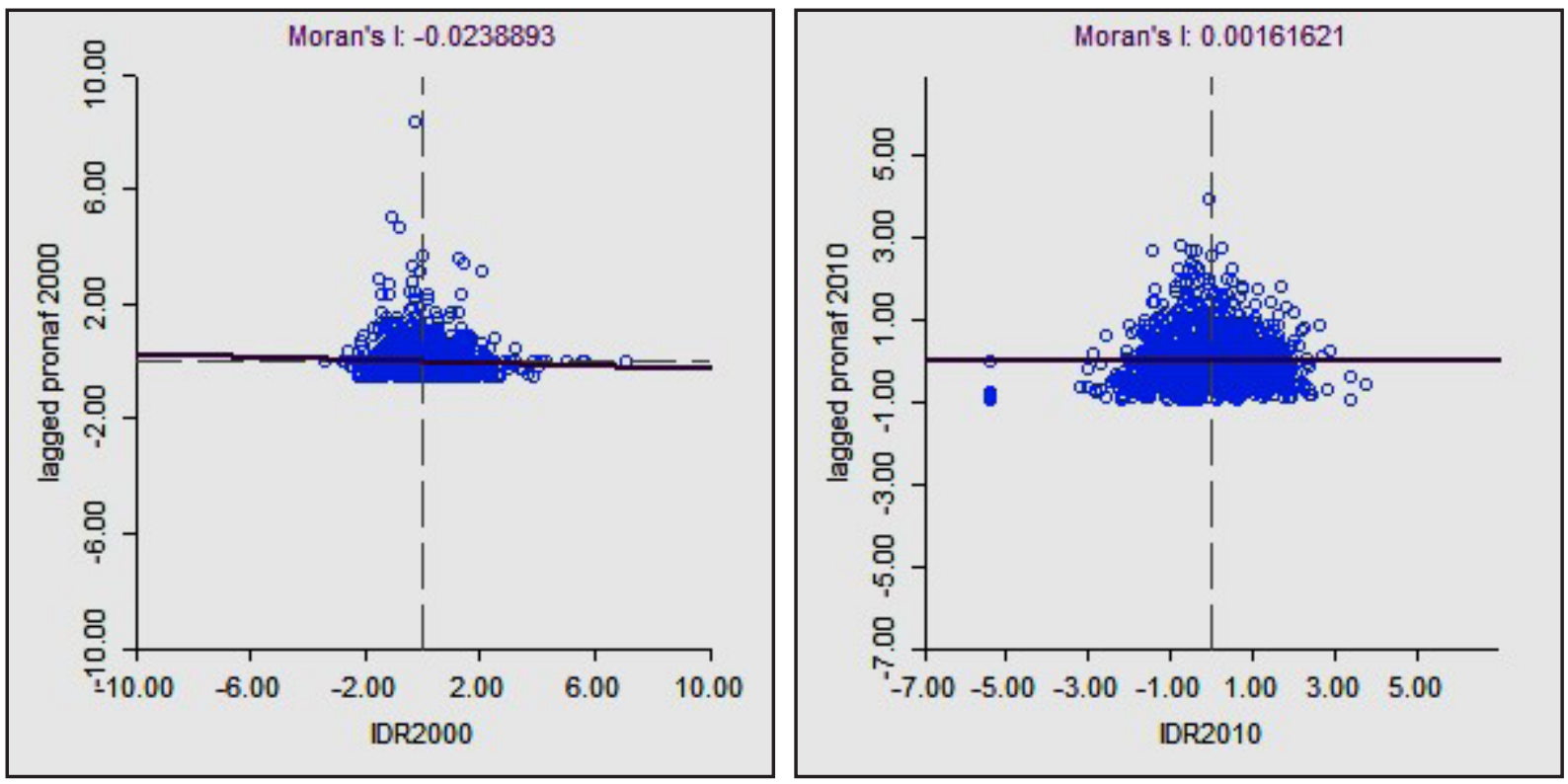

Fonte: Elaboração própria, a partir do cálculo do I de Moran Bivariado.

Para o ano 2000, o índice foi negativo e bastante próximo de zero (-0.0238), indicando uma correlação negativa entre o IDR e a distribuição dos recursos do PRONAF nos municípios do Nordeste. Para 2010, o índice é positivo, porém ainda mais próximo de zero (0.0016). Os resultados da análise global podem indicar que grande parte dos municípios não se mostraram significantes estatisticamente na relação espacial entre o IDR e os recursos do PRONAF. Porém, por ser um indicador global, é possível que o I de Moran Global venha mostrar que não há correlação nos dados, "enquanto existe uma forte correlação em diferentes partes da área de estudo", como afirma Matkan (2013, p. 198), sendo necessária uma análise local. Assim, em relação às variáveis aqui analisadas, o Índice Local Bivariado mostrará os padrões espaciais nos municípios significativos estatisticamente.

\subsection{Análise a partir do Índice de Moran Local Bivariado}

Esta segunda parte da análise apresenta os resultados encontrados a partir do cálculo do I de Moran Local Bivariado, pelo qual podem ser visualizadas as aglomerações espaciais, cluster e outliers. A seguir, nas Figuras 5 e 6 e nas Figuras 7 e 8, são apresentados os resultados do I de Moran Local Bivariado com os mapas de cluster e significância para 2000 e 2010, respectivamente, conhecidos também como mapas de LISA (Indicadores Locais de Associação Espacial). Para o ano de 2000, não foi possível obter informações sobre os valores do PRONAF para todos os municípios; assim, visualizando os mapas de 2000 e 2010, será possível perceber o aumento dos aglomerados significantes em 2010, já que, para esse ano, as informações abarcaram quase a totalidade dos municípios. 
Figuras 5 e 6 - Mapas de LISA Bivariados para IDR e PRONAF em 2000

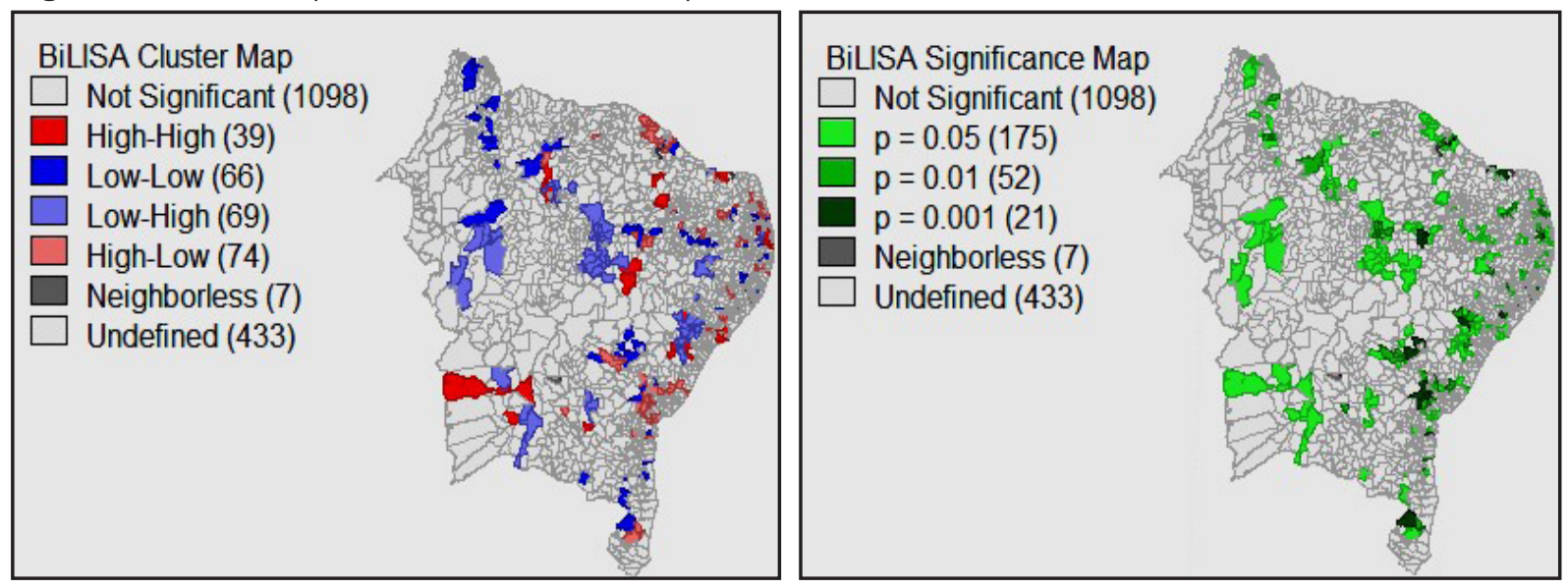

Fonte: Elaboração própria, a partir do cálculo do I de Moran Local Bivariado.

Por meio da análise Bivariada Local, podem ser visualizados os clusters Alto-Alto (AA) e Baixo-Baixo (BB) e outliers Alto-Baixo ( $A B$ ) e Baixo-Alto (BA), assim como sua significância estatística. Para o ano 2000, é possível observar alguns pequenos aglomerados do tipo AA nas áreas litorâneas, em sua maioria, mais próximos das capitais que do interior do mapa. Um destes aglomerados é formado por quatro municípios no litoral do Rio Grande do Norte. Um outro aglomerado é composto de oito municípios ao redor da capital João Pessoa e, logo abaixo, há um aglomerado com quatro municípios de Sergipe e um de Alagoas.

Ainda, para o ano de 2000, no interior do mapa há um aglomerado próximo à capital Teresina, formado pela própria capital e mais cinco municípios. Há também alguns municípios AA no extremo oeste baiano. Em relação aos aglomerados BB, eles aparecem mais no interior do mapa. É possível observar que os maiores aglomerados são do tipo BA, ou seja, municípios com IDR baixo, cujos vizinhos tem PRONAF alto; e os AB, municípios com IDR alto com vizinhos de PRONAF baixo. Além disso, a correlação negativa é a mais significativa estatisticamente, com p-valor 0,001 nos aglomerados AB e BA, como mostra o mapa de significância nas partes pintadas de verde-escuro.

De forma geral, nos resultados para 2000, nota-se que os valores mais elevados de IDR e PRONAF estão nas regiões litorâneas, indo ao encontro dos resultados encontrados por Michalek e Zarnekow (2011), que identificaram, em regiões da União Europeia, os maiores índices de desenvolvimento rural mais próximos aos centros urbanos e os menores em regiões mais afastadas e com pior infraestrutura local. Alguns outros valores significativos estão em localidades específicas da região, como nas proximidades do São Francisco, que, segundo Oliveira Filho et al. (2014), é uma região considerada polo de desenvolvimento em meio ao semiárido, devido ao cultivo irrigado, principalmente de frutas para exportação.

Em relação à autocorrelação local para o IDR e o PRONAF em 2010, as Figuras 7 e 8 mostram os mapas de LISA para as duas variáveis. Nota-se que há poucos municípios com informações faltantes sobre o PRONAF e há um aumento na quantidade de unidades com resultados significantes. 
Figuras 7 e 8 - Mapas de LISA Bivariados para o IDR e PRONAF em 2010

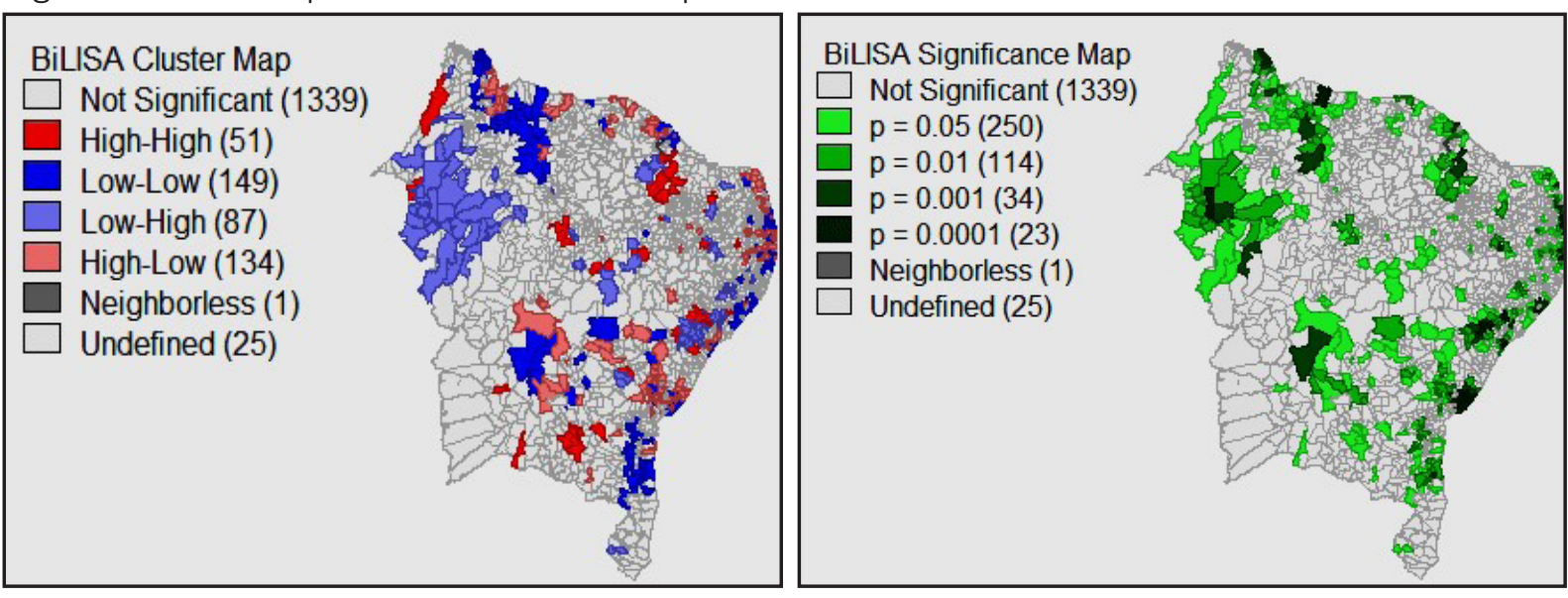

Fonte: Elaboração própria, a partir do cálculo do I de Moran Local Bivariado.

Para 2010, apesar de o índice global ter sido mais próximo de zero, é possível observar uma quantidade maior de aglomerações espaciais e com uma quantidade maior de municípios em cada quadrante. A maior quantidade de municípios poder vista no quadrante do tipo BB, em que municípios com baixo IDR são vizinhos de municípios com baixo PRONAF; e no quadrante $A B$, em que municípios com alto IDR são vizinhos de municípios com baixo PRONAF. É possível observar uma diferença na dinâmica espacial de 2000 para 2010. Em 2010, há uma redução dos clusters AA nas bordas do mapa (regiões litorâneas e mais próximas das capitais), em relação a 2000, e surgem mais clusters dos tipos BB e AB.

Para 2010, quase todas as capitais estão cercadas por aglomerados de municípios BB, exceto São Luiz e João Pessoa, que estão cercadas por clusters AB. Tal contexto reflete sobre a mudança de cenário de 2000 para 2010, acerca da dinâmica das atividades rurais que perderam protagonismo nas regiões metropolitanas ou próximas, influenciada pela industrialização e urbanização dessas áreas (PEREIRA JUNIOR, 2015). Essa mudança, certamente, contribuiu para uma diminuição na demanda pelos recursos do PRONAF nessas localidades, resultando em aglomerados de municípios com características BB. O mapa de significância de 2010 mostra as partes em verde-escuro com p-valor de 0,0001 e 0,001 indicando alta significância, principalmente onde se encontram os clusters BB.

Nota-se que os estados com maiores números de municípios em aglomerações do tipo BA é Sergipe, com um aglomerado de pelo menos 15 municípios, e o Maranhão, com um enorme aglomerado de pelo menos 30 municípios do tipo BA; ou seja, municípios com baixo IDR vizinhos de municípios com elevado PRONAF. É possível notar também um grande cluster BB no Maranhão vizinho do cluster $A B$ que circunda a capital São Luís.

De modo geral, nota-se que o Maranhão é o estado que apresenta os valores mais baixos de IDR e PRONAF. Porém a predominância desses valores baixos não é isolada para este índice, outros indicadores no Maranhão estão abaixo dos outros estados brasileiros, como o IDH de 0,63 e a renda domiciliar per capita de R\$597,00, que é a menor do País (IBGE, 2017).

Os aglomerados do tipo AA são aqueles com menor número de municípios, ainda assim, é possível observar alguns que se destacam. Por exemplo, o maior deles, no Ceará, é composto por oito municípios, e outro é formado por cinco municípios dos estados de Sergipe e Alagoas. Além destes, há um cluster na microrregião centro sul da Bahia, formado por cinco municípios. 
Esses aglomerados estão localizados mais para o interior do mapa, ao contrário dos aglomerados AA em 2000. É possível que, com uma maior industrialização e urbanização das cidades próximas às capitais, a agricultura venha perdendo espaço como atividade econômica e, por essa razão, o IDR e o PRONAF apresentem resultados mais baixos, pois, como afirma Pereira Junior (2015), os complexos industriais e portuários estabelecidos em diversas cidades nordestinas, nas últimas décadas, sejam elas metropolitanas ou próximas, geraram alterações radicais na configuração espacial produtiva dessas localidades, como um fluxo migratório crescente e a conversão do uso da terra rural em urbana, por exemplo.

\section{CONSIDERAÇÕES FINAIS}

Considerando a importância das atividades rurais como meio de vida para grande parte das famílias nordestinas, não se esgotam as tentativas de entender a relação entre as políticas destinadas ao desenvolvimento rural e indicadores que buscam captar em alguma medida as condições de vida dessas populações rurais. Diante disso, essa pesquisa teve o objetivo de analisar a relação espacial entre o IDR e os recursos do PRONAF acessados pelos municípios nordestinos nos anos de 2000 e 2010.

Uma primeira consideração a ser feita em relação aos resultados é quanto aos valores do IDR. O valor médio do índice aumentou de 2000 para 2010, o que pode ser visto, a princípio, como um bom indicativo de que o IDR vem aumentando nos municípios. Porém os municípios com IDR acima da média estão ainda bastante concentrados, como visto nos mapas de distribuição. Além disso, o valor máximo do IDR diminuiu, podendo indicar que, apesar da expansão agrícola no Nordeste e uma maior atuação da região nas exportações agrícolas nos últimos anos, como afirma Miranda (2012), as condições socioeconômicas e ambientais da região podem não ter acompanhado esse crescimento.

Em relação aos padrões de distribuição espacial entre o IDR e a distribuição do dos recursos do PRONAF, foi possível observar, principalmente no mapa de LISA 2010, que a maioria dos municípios onde foi identificada relação espacial, sejam clusters, sejam outliers, está mais próxima das extremidades do mapa. No território semiárido, por sua vez, localizado mais no interior do mapa e que possui grande necessidade de apoio ao desenvolvimento rural, não foi possível detectar relação espacial estatisticamente significativa entre o IDR e o PRONAF.

Além disso, existe uma notável diferença na distribuição espacial das variáveis de 2000 para 2010, como o surgimento de clusters baixo-baixo em 2010, compostos por diversos municípios próximos das capitais. Essa mudança pode indicar que áreas mais próximas do litoral nordestino perderam seus aspectos rurais no processo de industrialização, justificando, assim, o deslocamento dos aglomerados de municípios com IDR acima do terceiro quartil, os quais diminuíram nas bordas do mapa e se espalharam em direção ao interior da região em 2010, incluindo localidades onde a produção agrícola vem tendo destaque, como no Oeste Baiano e na região do vale do São Francisco.

O Índice de Moran Global Bivariado apresentou resultado próximo de zero para 2000 como também para 2010, o que a princípio poderia indicar uma correlação espacial pequena entre o PRONAF, que é uma das principais políticas de desenvolvimento rural e Índice de Desenvolvimento Rural nos anos estudados. Porém, na análise local, foi possível identificar diversos clusters em que as duas variáveis aparecem correlacionadas espacialmente. 
Dessa forma, entende-se a importância de considerar a localização espacial dos municípios na investigação da relação entre as políticas destinadas ao desenvolvimento rural e indicadores de desenvolvimento rural, pois, como já observado, é mais provável que os municípios, principalmente contíguos, exerçam alguma influência em relação aos seus vizinhos e sofram influências destes, a partir das relações intermunicipais e considerando seu grau de proximidade. É possível inferir, portanto, que o nível de desenvolvimento rural de um município e seu acesso aos recursos do PRONAF dependem, em alguma medida, das suas relações com os municípios vizinhos. Nesse sentido, faz-se necessário, ainda, entender as relações formais e informais estabelecidas entre os municípios que, por sua vez, exercem influência nos seus vizinhos, reproduzindo, como no caso dos municípios do Nordeste, uma condição predominante de baixo nível de desenvolvimento rural.

\section{REFERÊNCIAS}

ALMEIDA, E. S. Curso de Econometria Espacial Aplicada. Piracicaba: USP, 2004.

ANSELIN, L.; SYABRI, I.; KHO, Y. GeoDa: an introduction to spatial data analysis. Geographical analysis, Illinois, v. 38, n. 1, p. 5-22, 2006.

ANSELIN, L.; SYABRI, I.; SMIRNOV, O. Visualizing multivariate spatial correlation with dynamically linked windows. Urbana: REAL/University of Illinois, 2002.

ARAÚJO, J. A.; VIEIRA FILHO, J. E. R. Análise dos impactos do Pronaf na agricultura do Brasil no período de 2007 a 2016. Rio de Janeiro: IPEA, 2018.

BASILE, R. Regional economic growth in Europe: a semiparametric spatial dependence approach. Papers in Regional Science, [s.l.], v. 87, n. 4, p. 527-44, 2008. Disponível em: https://doi.org/10.1111/j.14355957.2008.00175.x. Acesso em: 14 maio 2018.

BATISTA, H. R.; NEDER, H. D. Efeitos do Pronaf sobre a pobreza rural no Brasil (2001-2009). Revista de Economia e Sociologia Rural, Brasília, v. 52, supl. 1, p. 147-66, 2014. Disponível em: http://www.scielo. br/scielo.php?pid=S0103-20032014000600008\&script=sci_arttext\&tlng=es. Acesso em: 20 jun. 2018.

CASTRO, C. N.; RESENDE, G. M.; PIRES, M. J. S. Avaliação dos impactos regionais do Programa Nacional de Agricultura Familiar (PRONAF). Brasília: IPEA, 2014. [Texto para Discussão, n. 1974].

COMITÊ DE OXFORD PARA ALÍVIO DA FOME [OXFAM]. Inequalities in Brazil: the divide that unites us. Setembro, 2017. Disponível em: https://www.oxfam.org/en/research/distance-unites-us-overviewbrazilian-inequalities. Acesso em: 14 fev. 2018.

CORRÊA, V. P.; SILVA, F. F.; NEDER, H. D. Construção de um índice de desenvolvimento rural: resultados para as regiões Nordeste e Sul do Brasil. In: CONGRESSO DA SOCIEDADE BRASILEIRA DE ECONOMIA, ADMINISTRAÇÃO E SOCIOLOGIA RURAL, 46., 20-23 jul. 2008, Rio Branco. Anais [...]. Rio Branco: Sober, 2008.

DAMASCENO, N. P.; KHAN, A. S.; LIMA, P. V. P. S. O impacto do Pronaf sobre a sustentabilidade da agricultura familiar, geração de emprego e renda no Estado do Ceará. Revista de Economia e Sociologia Rural, Brasília, v. 49, n. 1, p. 129-56, 2011.

DETHIER, J. J.; EFFENBERGER, A. Agriculture and development: a brief review of the literature. Economic Systems, [s.l.], v. 36, n. 2, p. 175-205, 2012. Disponível em: https://www.sciencedirect.com/science/article/ abs/pii/S0939362512000271. Acesso em 2 jun. 2018. 
FERNANDES, F. F. Programa Nacional de Fortalecimento da Agricultura Familiar (PRONAF): análise da distribuição dos recursos entre 1999 e 2009. 2011. Dissertação (Mestrado em Ciências Sociais Aplicadas) - Universidade Federal de Uberlândia, Uberlândia, MG, 2011.

GRISA, C.; WESZ JUNIOR, V. J.; BUCHWEITZ, V. D. Revisitando o Pronaf: velhos questionamentos, novas interpretações. Revista de Economia e Sociologia Rural, Brasília, v. 52, n. 2, p. 323-36, 2014.

IBGE. Maranhão - Panorama. IBGE, Rio de Janeiro, 2017. Disponível em: https://cidades.ibge.gov.br/ brasil/ma/panorama. Acesso em: 12 jan. 2019.

KAGEYAMA, A. Desenvolvimento rural: conceito e medida. Cadernos de Ciência \& Tecnologia, Campinas, v. 21, n. 3, p. 379-408, 2004. Disponível em: http://www.sober.org.br/palestra/12/120506.pdf. Acesso em: 10 jun. 2017.

LESAGE, J. P.; FISCHER, M. M. Spatial growth regressions: model specification, estimation and interpretation. Spatial Economic Analysis, [s.I.], v. 3, n. 3, p. 275-304, 2008. doi: https://doi. org/10.1080/17421770802353758

LINS, J. G. M. G.; LOURES, A. R.; SILVA, M. V. B.; LOMBARDI FILHO, S. C. Análise espacial da evolução do índice de desenvolvimento humano nos municípios da Região Nordeste. Revista Economia e Desenvolvimento, [s.l.], v. 14, n. 1, p. 81-89, 2015. Disponível em: https://periodicos.ufpb.br/index.php/economia/article/ view/27514/14796. Acesso em: 24 set. 2019.

MATKAN, A. A.; MOHAYMANY, A. S.; SHAHRI, M.; MIRBAGHERI, B. Detecting the spatial-temporal autocorrelation among crash frequencies in urban areas. Canadian Journal of Civil Engineering, Ottawa, v. 40, n. 3, p. 195-203, 2013.

MELO, C. O.; PARRÉ, J. Luiz. Índice de desenvolvimento rural dos municípios paranaenses: determinantes e hierarquização. Revista de Economia e Sociologia Rural, Rio de Janeiro, v. 45, n. 2, p. 329-65, 2007. Disponível em: https://www.scielo.br/j/resr/a/cBDhTKTHGdPfwGJ9zYdQpCF/?lang=pt\&format=pdf. Acesso em: 20 nov. 2018.

MICHALEK, J.; ZARNEKOW, N. Application of the rural development index to analysis of rural regions in Poland and Slovakia. Social indicators research, [s.l.], v. 105, n. 1, p. 1-37, 2011. Disponível em: https:// www.jstor.org/stable/41409399. Acesso em: 12 set. 2018.

OLIVEIRA FILHO, S. F. S.; MELO, A. D. S.; XAVIER, L. F.; SOBEL, T. F.; COSTA, E. D. F. Adoção de estratégias para redução de riscos: identificação dos determinantes da diversificação produtiva no Polo PetrolinaJuazeiro. Revista de Economia e Sociologia Rural, Brasília, v. 52, n. 1, p. 117-38, 2014. doi: https://doi. org/10.1590/S0103-20032014000100007.

ORGANIZAÇÃO PARA A COOPERAÇÃO E DESENVOLVIMENTO ECONÔMICO [OCDE]. A new rural development paradigm for the 21st century: a toolkit for developing countries. Paris: OECD, 2016. Disponível em: http://www.oecd-ilibrary.org/development/a-new-rural-development-paradigm-for-the21st-century_9789264252271-en. Acesso em: 15 dez. 2018.

PEREIRA JUNIOR, E. Dinâmicas industriais e urbanização no Nordeste do Brasil. Mercator - Revista de Geografia da UFC, Fortaleza, v. 14, n. 4, p. 63-81, 2015. doi: https://doi.org/10.4215/RM2015.1404.0005. Acesso em: 18 jan. 2019.

RODRÍGUEZ-POSE, A.; HARDY, D. Addressing poverty and inequality in the rural economy from a global perspective. Applied Geography, London, v. 61, p. 11-23, 2015. 
SILVA, F. F. Distribuição de crédito para agricultura familiar: um estudo do PRONAF a partir de um indicador de desenvolvimento rural. 2006. Dissertação (Mestrado em Ciências Sociais Aplicadas) - Universidade Federal de Uberlândia, Uberlândia, MG, 2006.

SILVA, L. D. C.; FARIAS SOUZA, W. P. S.; SILVA, M. V. B. Análise espacial da extrema pobreza na região Nordeste: uma aplicação para os municípios. In: ENCONTRO DE ECONOMIA BAIANA, 9., 19 e 20 setembro de 2013, Salvador, Bahia. Anais [...]. Salvador: UFBA, 2013. Disponível em: https://www.researchgate.net/ publication/307632800. Acesso em: 20 dez. 2019.

STEGE, A. L.; PARRÉ, J. L. Desenvolvimento rural nas microrregiões do Brasil: um estudo multidimensional. Revista Teoria e Evidência Econômica, [s.I.], v. 17, n. 37, p. 160-193, 2011. Disponível em: http://seer.upf. br/index.php/rtee/article/view/4227/2717. Acesso em: 15 jan. 2019.

TAE-HWA, K.; SEUNG-RYONG, Y. A. N. G. Construction of the rural development index: the case of Vietnam. Journal of Rural Development/Nongchon-Gyeongje, [s.l.], v. 39, n. 39, p. 113-42, 2016. Disponível em: https://ageconsearch.umn.edu/record/251931/. Acesso em: 18 fev. 2019.

VIEIRA FILHO, J. E. R. Distribuição produtiva e tecnológica dos estabelecimentos agropecuários de menor porte e gestão familiar no Brasil: ganhar tempo é possível? Brasília: CGEE, 2013.

\section{Sobre os autores:}

Geizibel Lopes Rodrigues: Mestre em Administração e Desenvolvimento Rural pela Universidade Federal Rural de Pernambuco (UFRPE). Graduada em Administração pela UFRPE, Unidade Acadêmica de Serra Talhada (UAST). E-mail: geizibellopes@gmail.com, Orcid: http://orcid.org/0000-0002-9427-5632

Diego Firmino Costa da Silva: Doutor, mestre e graduado em Economia pela Universidade Federal de Pernambuco (UFPE).E-mail: diegofirmino@gmail.com, Orcid: http://orcid.org/0000-0001-8819-7546 
